\title{
21. An Experimental Study of Stereotaxic Destruction on the Cat Brain by Intense Focused Ultrasound
}

\author{
Minoru Ishit, T. TAKeuchi, S. Katsumi, \\ S. KIKUCHI and K. TANAKA \\ Juntendo Univ. School of Medicine, Department of 2nd Surgery
}

In this experiment, concave barium titanate $80 \mathrm{~mm}$ in diameter, frequency of 1 megacycle per second was used as an ultrasonic transducer. Intensity of ultrasonic focused area was measured by means of thermistor sonde and we were able to calibrate that the focal averrage intensity was $320 \mathrm{w} / \mathrm{cm}^{2}$ and the peak intensity was $1400 \mathrm{w} / \mathrm{cm}^{2}$.

In order to make easy the ultrasonic irradiation procedure, we made the plastic pan connecting to the transducer and pan hole covered with thin rubber membrane, then the pan was filled with degassed water. Following craniotomy the focused ultrasound irradiated in the cat brain through the rubber membrane and the dura mater. This method was very easy and successful.

In our series of experiments we discovered that the size of ultrasonic lesion was able to be produced from $1 \times 2 \mathrm{~mm}$ to $10 \times 8 \mathrm{~mm}$.

Histological findings showed pan necrosis within the focal region, which was sharply demarcated from the surrounding healthy tissue, but no other changes along the pathway. Furthermore, it is characterized by intact capillaries in the ultrasound lesions. Following these results, it is reasonable that the focused ultrasound is a successful method for the study of the neurophysiology and for application in the neurosurgery.

While the location of the targets has been generally based upon the JasperMarsan's atlas of cat brain, but for the determination of more accurate location, we tried to take ventriculography of cat using a new diffusible $\mathrm{X}$-ray contrast medium "CONRAY" (Methylglucamine Methylamate 60\%). According to this ventriculogram of cat and Jasper-Marsan's atlas, we were able to determine the stereotaxic point more accurately.

\section{Discussing the Blood-Brain Barrier in Tissue Culture}

-The Function Morphology of Astrocytes and Endothelial Cells-

Yohji Hara, Jun Tominaga, and Tsuneyuki Nakazawa

Department of Neuropsychiatry, Keio Gijiku University

School of Medicine

The astrocytes and endothelial cells cultivated from cerebcllum, meninges anc

$$
-160-
$$

